\title{
PROGRAMA DE SUPERACIÓN PROFESIONAL PARA PROFESORES UNIVERSITARIOS DE IDIOMA INGLÉS
}

\author{
Professional overcoming program for english language university \\ teachers
}

\section{Caridad Abreu*}

\section{RESUMEN}

El objetivo del presente artículo es describir las características, requisitos y componentes que debe tener un programa de superación profesional para preparar a profesores universitarios de idioma inglés en la dirección del proceso de enseñanzaaprendizaje en la modalidad semipresencial. Autores como: Añorga (1997), Arroyo (2001), Carretero (2002), Berges (2003), Gallardo (2004), Lombana (2005), Romero (2007), Sánchez (2013), entre otros sirvieron de referentes teóricos, sin embargo, a pesar del incuestionable valor de los criterios emitidos por los autores consultados, no se constató la existencia de investigaciones relacionadas con la superación profesional para los profesores de idioma inglés en la modalidad semipresencial. Se procedió de acuerdo con los requerimientos de la investigación-acción participativa.La muestra estuvo conformada por 20 profesores del Departamento de Lenguas Extranjeras de la Universidad Central Marta Abreu de Las Villas ,que imparten Idioma Inglés en carreras técnicas .Para la recolección de datos se aplicó el análisis de documentos, observación, entrevistas y encuestas, para registrar la información se utilizaron notas de campo y transcripciones de las entrevistas.El Programa de Superación Profesional se distingue por asumir el asesoramiento colaborativo como forma de superación profesional. Como conclusión se constató un aumento en el desarrollo de los niveles de preparación de los profesores que les permitió potenciar la reflexión, la metacognición y la toma de decisiones a partir de los conocimientos recibidos durante el programa de superación profesional y, en consecuencia, dirigir el proceso de enseñanza - aprendizaje de la disciplina inglés en la modalidad semipresencial.

Palabras claves: Superación Profesional, asesoramiento colaborativo, idioma inglés, modalidad semipresencial.

\footnotetext{
* Doctora en Ciencias Pedagógicas. Profesora Titular. Profesora de Idioma Inglés a tiempo Integral en la Universidad Óscar Ribas. Luanda -Angola. Correo electrónico: carypa2013@gmail.com 


\begin{abstract}
The objective of this article is to describe the characteristics, requirements and components that a professional improvement program must have in order to prepare university teachers of English language in the direction of the teaching-learning process in the blended modality. Authors such as, Añorga (1997), Arroyo (2001), Berges (2003), Gallardo (2004), Lombana (2005), Romero (2007), Sánchez (2013), among others, served as theoretical references, without However, despite the unquestionable value of the criteria issued by the authors consulted, the existence of research related to professional development for English language teachers in the blended modality was not confirmed. We proceeded according to the requirements of the participatory action research. The sample consisted of 20 professors from the Department of Foreign Languages of the Central University Marta Abreu de Las Villas, who taught English in technical careers. applied the analysis of documents, observation, interviews and surveys, to record the information field notes and transcripts of the interviews were used. The Professional Improvement Program is distinguished by assuming collaborative advice as a way of professional improvement. In conclusion, there was an increase in the development of teacher preparation levels that allowed them to enhance reflection, metacognition and decision making based on the knowledge received during the professional development program and consequently, direct the process of teaching - learning of the English discipline in the blended modality.
\end{abstract}

Key words: Professional Development, collaborative assessment, English language, blended modality.

\title{
Introducción
}

La aplicación de la modalidad semipresencial ha representado un gran reto y un desafío para los profesores universitarios de la disciplina Idioma Inglés (Corona, 2006) dadas las características del proceso de enseñanza-aprendizaje de esta disciplina que imparten, y la ausencia de una experiencia anterior en la enseñanza del idioma inglés con una modalidad semipresencial a diferencia de otras disciplinas que se estudian en los centros de educación superior de Cuba (Abreu, 2007).

Esta situación ha generado insuficiencias en los profesores de Idioma Ingles que imparten esta disciplina en la modalidad semipresencial ya que no consiguen desarrollar la autonomía ni la independencia cognoscitiva en el estudiante universitario, 
cuestión esta que repercute en la calidad del proceso de enseñanza-aprendizaje del mismo.

Por otro lado, en la actualidad, independientemente de la variedad de ofertas de superación postgraduada para los docentes, se evidencia la carencia de una forma de superación que pudiera contribuir a la preparación de los profesores de idioma inglés para dirigir el proceso de enseñanza-aprendizaje en la modalidad semipresencial.

En este sentido, múltiples son los autores que han abordado la superación profesional y los enfoques referidos a ella. Entre estos autores se encuentran: Sánchez (2013), Romero (2007), Gómez (2007), Lombana (2005), Gallardo (2004), Berges (2003), Arroyo (2001), Añorga (1997), entre otros; sin embargo, a pesar del incuestionable valor de los aportes de los referidos autores, no se ha constatado la existencia de trabajos relacionados con la superación profesional para los profesores de idioma inglés en la dirección del proceso de enseñanza-aprendizaje en la modalidad semipresencial.

Lo anterior llevó a la búsqueda de soluciones viables dirigidas a la superación profesional de este docente en correspondencia con las exigencias de la modalidad semipresencial. La misma debe ser organizada a través de formas de superación orientadas al perfeccionamiento del modo de accionar del docente a partir de sus vivencias y así permitir la elaboración del conocimiento, la reflexión y el análisis mediado por la colaboración y la toma de decisiones (Abreu, 2008).

En este sentido la búsqueda de formas de superación que propicien la reflexión, la metacognición y la toma de decisiones, imprescindibles en momentos de cambio, condujo al estudio de trabajos de autores como Peralta (2006), Monereo y Pozo (2005), Nieto (2005), entre otros, quienes abordan el asesoramiento psicopedagógico con diferentes fines.

De especial interés han sido el tratamiento de las diferentes formas del asesoramiento psicopedagógico abordadas por Carretero (2002), en particular el asesoramiento colaborativo; y los criterios expuestos por Escudero y Moreno (1992) para desarrollarlo toda vez que se ajustan a las características del tipo de superación a que se 


\section{Caridad Abreu}

Telos Vol. 21, No. 1 (2019). 114-140

aspira.

El asesoramiento colaborativo se caracteriza por el diálogo, la colaboración, el intercambio entre las partes implicadas (asesor-asesorados), y se erige de manera flexible (Carretero, 2002). Desde esta perspectiva el asesoramiento colaborativo puede ser considerado el requisito indispensable para desarrollar hábitos y conductas que propicien una enseñanza reflexiva, manifestado en la habilidad para discutir y criticar estrategias propias de resolución de problemas y modelos de conocimiento (López, 1997).

Desde esta visión, se asume la superación profesional bajo un enfoque que implica procesos de aprendizaje diversos desde el análisis y la reflexión sobre la práctica, hasta el dominio de nuevos contenidos y habilidades a partir del conocimiento pedagógico;la misma debe ser organizada a través de formas de superación orientadas al perfeccionamiento del modo de accionar del docente a partir de sus vivencias y así permitir la elaboración del conocimiento, la reflexión y el análisis mediado por la colaboración que se propone (Abreu, 2008).

En este orden de ideas, se presenta el Programa de Superación Profesional para profesores de idioma inglés, el cual se distingue por asumir el asesoramiento colaborativo como forma de superación para lograr la preparación del profesor universitario de Idioma Inglés en la dirección del proceso de enseñanza-aprendizaje de la disciplina inglés en la modalidad semipresencial, el mismo se fundamenta en el enfoque HistóricoCultural de Vigotsky y sus seguidores con procedimientos psicológicos y pedagógicos presentes en el asesoramiento colaborativo.

Constituye objetivo del presente artículo describir las características, requisitos y componentes que debe tener un programa de superación profesional para preparar a profesores universitarios de idioma inglés en la dirección del proceso de enseñanza-aprendizaje en la modalidad semipresencial,para lo cual se estructuró en seis componentes:1)introducción, 2)aspectos metodológicos; 3) fundamentación teórica del programa de superación profesional; 4) características, requisitos y componentes del 
programa de superación profesional. 5)resultados de la aplicación del programa; y 6) conclusiones; desarrollo del cual se presenta a continuación.

\section{Aspectos metodológicos}

Durante el proceso investigativo se procedió de acuerdo con los requerimientos de la investigación-acción participativa, la cual se caracteriza por un conjunto de normas y procedimientos metodológicos que consideran la colaboración entre los participantes, lo que a su vez propicia el logro de conocimientos colectivos para transformar determinada realidad, es un proceso de búsqueda de conocimientos, pero en este caso los conocimientos se adquieren de forma colectiva (Rodríguez, et al 2004)

En correspondencia con la Investigación-Acción y siguiendo el criterio general de Lagardera (1998), el proceso realizado discurrió mediante cuatro fases cuyo contenido ha sido adaptado en función de la presente investigación. Estas fases quedan expresadas como sigue:

Fase I Preparación

Fase II. Diagnóstico de necesidades

Fase III. Estructuración del programa de superación

Fase IV. Plan de acciones para perfeccionar el programa de superación

Desde esta perspectiva, el profesor investiga sobre su práctica en un proceso que ayuda a conformar un pensamiento teórico más abarcador y totalizador de la realidad, para incidir en la práctica y transformarla; en una dinámica en la que conocer y transformar, conforman una unidad.

Por otro lado, el profesor tiene, en la investigación-acción participativa uno de sus referentes principales, ya que se trata de una propuesta orientada de manera intencional a brindar conocimientos a las personas para que puedan cambiar la forma de pensar y, en consecuencia, la de actuar a partir de las alternativas de solución de sus contradicciones, posibilitando su autodesarrollo (Alonso, et al, 2004)

Teniendo en cuenta el objetivo de este trabajo, se optó por una muestra de 20 


\section{Caridad Abreu}

Telos Vol. 21, No. 1 (2019). 114-140

profesores del Departamento de Lenguas Extranjeras de la Universidad Central Marta Abreu de Las Villas, que imparten Idioma Inglés en las Carreras Técnicas. Para la recolección de datos se aplicó el análisis de documentos, observación, entrevistas y encuestas, para registrar la información se utilizaron notas de campo y transcripciones de las entrevistas.

Para llevar a cabo el diagnóstico de las necesidades de superación de los profesores de la disciplina Idioma inglés, se elaboraron diferentes instrumentos en correspondencia con los métodos: una guía para el análisis de documentos, una guía para la entrevista grupal en profundidad y una encuesta de autoevaluación

El análisis de documentos se realizó con el objetivo de conocer sobre lo que está estipulado en las reglamentaciones existentes relacionadas con la modalidad semipresencial y el tratamiento que recibe la misma en función de los cambios en la dirección del proceso de enseñanza-aprendizaje.

La entrevista grupal en profundidad fue aplicada al grupo de profesores que conforman la muestra con el objetivo de explorar la percepción de estos sobre la modalidad semipresencial. La entrevista, permitió obtener datos sobre el problema determinado, a partir del cual se estableció una lista de temas, en relación con los que se focaliza la entrevista, quedando a la libre discreción del entrevistador el posible sondeo de razones y motivos, así como la ayuda para establecer determinado factor, pero sin sujetarse a una estructura formalizada de antemano.

Mientras que la encuesta, permitió conocer las opiniones y valoraciones que sobre determinados asuntos poseen los sujetos seleccionados en la muestra, se utilizó para conocer los factores o causas que han generado un fenómeno, la valoración que hacen los sujetos y las dificultades que se afrontan en el desarrollo de la tarea.

La interpretación de la información obtenida se efectuó en función de los objetivos propuestos en la investigación, el criterio seguido fue organizar la información dándole sentido a través de la selección de los datos pertinentes para su integración en esquemas conceptuales. Al finalizar cada sesión del grupo de trabajo, 
siguiendo a Rodríguez, et al (2004), se elaboró un análisis preliminar que contenía un resumen de los hallazgos, interpretaciones, y posibilidad de modificaciones.

Luego fue empleada la técnica de análisis de contenido, definida como una técnica destinada a formular inferencias reproducibles y válidas y que pueden aplicarse a ese contexto para la información de las comunicaciones escritas orales y visuales, que su marco de referencia -al decir de Serrano (1999)- radica en considerar los datos tal y como se comunican al analista, el contexto de los datos, la forma en que el conocimiento del analista lo obliga a divisar la realidad, la inferencia como tarea intelectual, la validez como criterio seguro de éxito.

En este sentido fueron asumidas tres etapas consecutivas y recursivas: la etapa de preanálisis, la etapa de codificación y la etapa de categorización. En la primera fue organizado el material según los objetivos de la investigación. En la segunda fue analizado el corpus sobre la base de los objetivos de la investigación, se operaron los datos brutos a datos útiles. En la tercera etapa se organizaron y clasificaron las unidades a partir de categorías y subcategorías obtenidas sobre la base de los criterios de diferenciación para obtener una visión condensada de los datos trabajados.

\section{Discusión de los resultados del diagnóstico}

Los resultados obtenidos del diagnóstico, se discutieron en un encuentro donde participó tanto el grupo de trabajo como el grupo de profesores que conforman la muestra de la investigación. En ese encuentro se presentaron los resultados y se determinaron las principales regularidades del diagnóstico realizado.

El análisis de los resultados permitió determinar los factores que obstaculizan la dirección del proceso de enseñanza-aprendizaje de la disciplina Idioma Inglés en la modalidad semipresencial; así como las necesidades de superación inmediatas de los docentes.

El interés por resolver los problemas que se venían presentando en el desarrollo del proceso de enseñanza-aprendizaje de la disciplina Inglés en la 


\section{Caridad Abreu}

Telos Vol. 21, No. 1 (2019). 114-140

modalidad semipresencial, la relación entre las necesidades inmediatas de superación y las exigencias para la dirección del proceso de enseñanza- aprendizaje de esta disciplina en esa modalidad, así como el conocimiento sobre algunos aspectos elementales relacionados con intervenciones psicopedagógica en contextos educativos motivaron a buscar recursos para solucionar los problemas presentados mediante la propuesta del programa de superación profesional que se presenta .

Como conclusión del intercambio se decidió organizar un nuevo encuentro con el objetivo de analizar la propuesta sobre la estructuración del programa, lo que correspondió a la siguiente fase del proceso investigativo.

\section{Fundamentación teórica que sustenta el Programa de Superación Profesional para} preparar a los profesores universitarios en la dirección del proceso de enseñanzaaprendizaje en la modalidad semipresencial.

El programa de superación se fundamenta en las ciencias filosóficas, sociológicas, psicológicas, pedagógicas y lingüísticas, que se presentan a continuación:

\section{Fundamentos Filosóficos del programa}

La concepción del programa de superación profesional para preparar al profesor universitario de la disciplina Idioma Inglés en la dirección del proceso de enseñanza- aprendizaje en la modalidad semipresencial responde a la concepción filosófica que parte de la cognoscibilidad del mundo referida por Vigostky (1978); en este caso, estrechamente relacionada con la pertinencia del problema en los marcos de las transformaciones educacionales relacionados con la implementación de un nuevo programa en la disciplina Idioma Inglés en la modalidad semipresencial.

Por otro lado, el materialismo dialéctico es el método general que guía al pensamiento y a la acción educativa, además ofrece una base teórica esencial para comprender el proceso del conocimiento en el hombre (González, 1999). 
Particular significación tiene en el programa que se presenta la teoría del conocimiento. Según Álvarez (1999) esta teoría explica cómo el conocimiento es el resultado de la interrelación dialéctica del sujeto y los objetos de la realidad, y de los sujetos entre sí, el papel que juega la práctica, así como la relación entre conocimiento y valoración.

Por otro lado, esta teoría ayuda a considerar tres aspectos: la transformación de la forma de pensar, la conformación del modo de actuación del profesor y la concepción de su superación mediante la preparación teórico- metodológica para dirigir el proceso de enseñanza- aprendizaje de la disciplina que imparte en la modalidad semipresencial.

En el primero se tiene en cuenta la dialéctica que se da entre sentido y significado donde la reflexión juega un papel principal; en el segundo, la estimulación que se hace desde el proceso de superación para desarrollar las posibilidades y potencialidades de estos profesionales, para reflejar objetivamente la realidad y valorarla, donde intervienen la reflexión, la metacognición; y en el tercero, el papel de la práctica en la obtención del conocimiento por parte del sujeto alcanza el modo deseado a partir de la valoración sobre su modo de actuar y la toma de decisiones sobre la base de un diálogo que es participativo, reflexivo y colaborativo.

Desde este referente el proceso de enseñanza-aprendizaje que se genera en el programa de superación tiene que concebirse como un elemento de carácter liberador, genuinamente dialéctico, lo que permite al sujeto que aprende desarrollar la reflexión, la metacognición y la toma de decisiones, favorecer la creatividad y llegar a convertirlo en protagonista de su momento histórico, con un gran sentido de solidaridad y responsabilidad ante el papel que desempeña como docente que se supera.

\section{Fundamentos sociológicos del programa}

La proyección en el programa de un docente concebido como agente que se comporta como factor de cambio en medio de las referidas transformaciones, implica 


\section{Caridad Abreu}

Telos Vol. 21, No. 1 (2019). 114-140

esenciales principios sociológicos que responden a los sujetos superados en cuanto a sus vínculos de interdependencia, y sus particularidades como grupo humano, así como su sistema de relaciones y el conjunto de fuerzas impulsoras de la actividad social presentes en la concepción del trabajo individual y colectivo con que acceden al conocimiento (Freire,1996).

La necesidad de enfocar las relaciones educativas teniendo en cuenta las características del asesoramiento colaborativo, es traducida a los marcos de la superación posgraduada como fenómeno educativo, dirigida al desarrollo y autodesarrollo del hombre, su formación integral, la socialización de los contextos y la formación creadora; todas ellas desde los presupuestos que se asumen acerca del carácter condicionado y determinado de la educación en medio del desarrollo del proceso histórico y de la actividad social y productiva en sí.(Carretero ,2002 )

Las bases sociológicas del programa explican el transcurso de las relaciones en un plano material que promueve el reto de la cognoscibilidad en las condiciones concretas (materiales y espirituales) de la sociedad actual; todo en busca del mejoramiento en la calidad de vida y el desarrollo sostenible. Por ello, la idea del docente que se transforma en función de las demandas sociales implica una concepción de la enseñanza durante el desarrollo del programa que enfatiza la búsqueda de alternativas abriendo espacios al cambio en medio de funciones latentes que pueden aparecer durante el proceso como parte del carácter dialéctico del método y de los factores que participan.

En este sentido se concuerda con Álvarez (1999) en que la esencia de la educación que se promueve como fenómeno social, tributa a una demanda ideológica que a su vez supone un sistema de relaciones donde los actores, conforman una estructura social en funcionamiento que eleva su nivel de preparación por medio de un proceso activo, creador, investigador y experimentador, el cual estimula la individualidad, a la vez que desarrolla un carácter flexible, espontáneo y orientador en un clima altamente colaborativo; necesario al docente que se supera. 


\section{Fundamentos psicológicos del programa}

En su concepción psicológica, a partir de las ideas de Vygotsky(1987),el programa se fundamenta en el enfoque Histórico-Cultural y las regularidades que éste establece acerca del desarrollo psíquico, asumiendo los principios que enfocan a la actividad como núcleo esencial de la formación y el desarrollo históricamente condicionado de la personalidad, vista ésta como producto más acabado del psiquismo humano, transcurriendo en un curso regido por leyes que se expresan en las tendencias de su desarrollo, dirigible y modelable en el proceso formativo a partir de la interacción entre los procesos internos y los factores externos que inciden en su educabilidad, determinando un tránsito del conocimiento hacia la práctica bajo la incidencia de afectos, motivaciones, sentimientos e intereses que determinan la conducta concreta del sujeto.

Rigiéndose por principios básicos referentes al determinismo dialéctico del desarrollo psíquico, así como el principio de la naturaleza social del desarrollo psíquico, el diagnóstico se convierte en la base fundamental desde la cual se proyecta la superación profesional del profesor universitario de la disciplina Idioma Inglés, teniendo en cuenta que tras sus expresiones cognitivas, emocionales y otras reacciones subyacen elementos de la psiquis sobre los cuales puede influirse en función de la superación. La mediación de la actividad, por tanto, interactúa con el contexto sociocultural, permitiendo potenciar la construcción interna de las funciones psíquicas superiores en un nivel marcadamente consciente.

Siguiendo a Vygotsky, (1987), y tomando en cuenta la zona de desarrollo actual del sujeto en el programa se enfrenta el reto de estimular las relaciones afectivo-cognoscitivas y volitivas en un contexto que motive sus necesidades individuales y sociales bajo los postulados del buen aprendizaje, visto como aquel que precede al desarrollo favoreciendo las formas de obtención del conocimiento y sus métodos por medio de procesos meta reflexivos. En este caso, se prevé que el docente sea quien realice el control sobre su propio proceso de desarrollo por medio de 


\section{Caridad Abreu}

Telos Vol. 21, No. 1 (2019). 114-140

determinados niveles de ayuda, convirtiéndose el grupo y el investigador (asesorados $\mathrm{y}$ asesor) en facilitadores de intercambios para desarrollar habilidades desde los propios procesos vivenciales del sujeto; todo a través de la reorganización del desarrollo de las funciones psíquicas en la zona de desarrollo próximo.

Desde dicho enfoque, el programa de superación se vincula directamente con varios problemas medulares de la Psicología Educativa, entre los cuales sobresalen, los fundamentos de la atención a las diferencias individuales y la teoría del aprendizaje como esencia de la dirección en la actividad cognoscitiva.

\section{Fundamentos Pedagógicos del programa}

El programa en correspondencia con la primera ley de la Pedagogía (Álvarez, 1999),reconoce la unidad dialéctica entre las influencias educativas que interactúan durante su puesta en práctica y propicia que se tome en cuenta como factor esencial la existencia de una cultura humanística de base en los docentes, potenciando las fortalezas existentes y cubriendo los espacios que donde se presenten deficiencias teóricas y metodológicas obstaculizadoras de una concepción del conocimiento y las habilidades en esta área del saber. Por ello, el carácter condicionado y a la vez condicionante de la educación constituye otro punto de apoyo fundamental en la propuesta.

El proceso de enseñanza-aprendizaje que se diseña en el programa responde a un conjunto de exigencias que contribuyen a la materialización de la educación en la modalidad semipresencial y al carácter activo, consciente significativo, acorde con las características del contexto educativo. Lo anterior evidencia el cumplimiento de leyes y principios que norman y regulan dicho proceso.

En tal sentido, en el programa ,siguiendo las ideas (Arroyo , 1999) se propicia el desarrollo de una serie de acciones cognitivas, de comunicación e interrelación personal, con la utilización de materiales complementarios que sirven de apoyo al docente que se supera con el objetivo de solucionar un problema u obtener determinados resultados De 
ahí que el modo de actuación que se aborda está relacionado con una función de la actividad pedagógica actual, que obedece en este caso, a un reto que la universidad le plantea al profesor de idioma inglés: la dirección del proceso de enseñanza-aprendizaje de la disciplina Idioma Inglés en la modalidad semipresencial.

Por tanto, se asume que se derivan implicaciones metodológicas que determinan la concepción, organización y desarrollo del programa, que se concretan y adoptan en dependencia de los objetivos a alcanzar, las necesidades y características de los asesorados, el tiempo disponible, entre otros factores.

\section{Fundamentos Lingüísticos del programa}

El programa de superación profesional para preparar a profesores universitarios en la dirección del proceso de enseñanza- aprendizaje de la disciplina Idioma Inglés en la modalidad semipresencial se sustenta en fundamentos lingüísticos al asumir una concepción sistémica del lenguaje y admitirlo como un sistema coherente al tener en cuenta la función comunicativa de este.

En el desarrollo del programa se toman en cuenta los procesos de comunicación, de diálogo, de intercambio Halliday, (1982), entre asesor-asesorados, asesorados-asesor donde el condicionamiento social de la lengua vale tanto para el habla individual como para el habla colectiva, donde según o referido autor, se pone de manifiesto la función comunicativa como la fundamental del lenguaje. De ahí la importancia de que en el programa los asesorados comprendan tanto por qué como el qué y el cómo.

Asumir una concepción sobre la superación que eduque y desarrolle al que se supera en la solución de problemas de la práctica profesional, ha permitido asumir principios de la dirección del proceso pedagógico propuesta por Addine (2002), dado el carácter holístico de su concepción, su actualidad y la congruencia con las ideas que se defienden en esta investigación. Los principios que se asumen son:

Principio de la vinculación de la educación con la vida, el medio social y el 


\section{trabajo.}

Según Addine (2002), este principio pondera la necesidad de dar solución a los problemas que provengan de la práctica laboral y establece la dependencia de la educación de los objetivos de la sociedad. La integración de la teoría y la práctica, el estudio y el trabajo, constituyen pilares de este principio.

De hecho, el programa se concibe partiendo de dar solución a dificultades presentadas por los docentes en la dirección del proceso de enseñanza-aprendizaje de la disciplina Idioma Inglés en la modalidad semipresencial mediante la preparación teórico-metodológica del docente para dirigir dicho proceso y se estructura con espacios para confirmar esta teoría en la práctica.

\section{Principio del carácter colectivo e individual de la educación de la personalidad y el respeto a esta.}

Este principio revela que el programa se desarrolla en un proceso donde cada miembro es portador de una individualidad, que da paso a una diversidad en el contexto. Uno de los aspectos más novedosos de esta propuesta descansa en este principio.

El empleo de procedimientos metacognitivos en el programa se proyecta para el mejoramiento profesional del docente como individuo. Ello, unido al trabajo en grupo y de forma colectiva moviliza el desarrollo del pensamiento y refuerza conductas, a partir de experiencias adquiridas en estas relaciones interpersonales, lo cual constituye una alternativa para dar tratamiento a la diversidad y la unidad educativa. Addine (2002)

\section{Principio de la unidad entre lo cognitivo y lo afectivo}

Este principio fundamenta el funcionamiento integrado de la personalidad. Para González Rey (1989); esta unidad representa uno de los niveles más complejos de funcionamiento de la personalidad e implica la formación de motivos. Por esta 
razón es objetivo en el programa activar la aparición de motivos intrínsecos a través del desarrollo de las formas de superación que lo conforman y el sistema comunicación diseñado, que a su vez promueva significatividad en el aprendizaje e implique a los docentes en la toma de decisiones desplegando para ello su actividad metacognitiva y creadora en un contexto de relaciones entre asesor- asesorado- asesor donde como resultado las expectativas de superación y necesidad de mejoramiento profesional sean mayor.

Principio de la unidad entre la actividad, la comunicación y la personalidad

En el programa se ponen en práctica actividades que propician la producción de ideas, desarrollo de la reflexión, la comunicación, la toma de decisiones en un ambiente de colaboración que facilitan el tránsito hacia niveles superiores de desarrollo sobre la base de una comunicación asertiva, democrática, en un clima de respeto y empatía mediante el tipo de actividad que se concreta en el debate grupal, así como el reforzamiento de la autoestima de los docentes lo cual ha de incidir en su implicación afectiva y conductual y en su desarrollo como personalidad.

\section{Características, requisitos y componentes del programa de superación profesional para profesores universitarios de idioma inglés la dirección del proceso de enseñanza-aprendizaje en la modalidad semipresencial.}

El Programa de Superación profesional para profesores universitarios de idioma Inglés se caracteriza por el diálogo, la colaboración, la interrelación y el intercambio entre las partes implicadas combinando los momentos individuales y colectivos, por la presencia de un asesor (docente que orienta, guía el desarrollo del programa) quien controla la marcha y la evaluación del proceso mediante la autoevaluación, la coevaluación y la heteroevaluación, además de dirigir el trabajo con los profesores más que intervenir sobre ellos.

El dialogo, es entendido una vía para el desarrollo de un pensamiento crítico, el cual se erige preferentemente en colaboración con el otro, en una relación pensada a 


\section{Caridad Abreu}

Telos Vol. 21, No. 1 (2019). 114-140

nivel horizontal (Torres,2004), es decir, una conversación en donde exista la igualdad y la confianza.

Por tanto, se debe generar un ambiente de empatía, de cordialidad que permita desarrollar un diálogo entre todos, donde se puedan expresar y defender las ideas y reflexiones, todo lo cual propicia la negociación de significados, y el surgimiento de actitudes racionales y críticas que se hacen presentes en toda conversación.

La colaboración, por su parte, es entendida, en opinión de Romero (2007) como un proceso de construcción social en el que cada individuo aprende más de lo que aprendería por sí mismo, debido a la interactividad con otros miembros de su grupo, por tanto, es un proceso necesario para obtener mejores resultados en el proceso de enseñanza aprendizaje en la superación profesional.

Es válido destacar, que la clave de este tipo de relación de colaboración exige la construcción de una comprensión compartida desde las interpretaciones y percepciones de todos los participantes, a partir de una reflexión negociada, que incluya las áreas de desacuerdo, contradicciones y conflictos, teniendo como objetivo el cambio donde se priorice la solución de tareas docentes como forma de enseñanza problémica de gran pertinencia, de interés colectivo e individual y sobre todo de interés social, para de esta forma lograr el mejoramiento para la solución de los problemas, la optimización, y el crecimiento personal(Nieto, 2005)

De esta forma se hace referencia a un tipo de interrelación entre las partes implicadas en el proceso de superación(asesor-asesorado), donde se comparten responsabilidades en el análisis de las necesidades, así como la formulación de objetivos, y la búsqueda de soluciones a los problemas que se presentan en la comunidad educativa.

Desde este punto de vista, se concibe un intercambio de ideas a partir de la relación entre los participantes y su forma de propiciar el acercamiento al conocimiento (Nieto, 2005), donde se interviene con la intención de mejorar la práctica educativa a 
partir de un proceso de indagación compartida sobre los problemas y las necesidades que afectan a la realidad educativa de los involucrados.

El programa se fundamenta, en la teoría Histórico-Cultural, donde se asume a la enseñanza como guía al desarrollo, y el proceso transcurre en un grupo en el cual se propician múltiples relaciones sociales, y se enriquecen, produciéndose nuevas necesidades, conocimientos, experiencias, lo que obviamente no implica desconocer las particularidades de los integrantes. Además, se desarrolla en la modalidad semipresencial mediante el uso de métodos que propician la socialización del conocimiento, el intercambio y la confrontación para producir el aprendizaje.

Es distintivo en el programa el asumir el asesoramiento colaborativo como forma de superación profesional y erigirse de forma flexible, buscar y facilitar la máxima participación e implicación del profesorado, establecer un clima relacional y un tono afectivo, favorecer la toma de decisiones, así como el desarrollo de procesos que permitan a los docentes reflexionar sobre su práctica, y repensar la práctica para generar otro nivel de conocimiento.

El programa se ha nutrido de tendencias psicológicas y pedagógicas que han proporcionado aportes significativos al proceso de formación del docente que se supera en un proceso donde se incentiva la reflexión, la construcción del conocimiento, la metacognición, la toma de decisiones y se generan aprendizajes colaborativos entre los docentes.

De esta manera, el mismo cumple con uno de sus requisitos esenciales, lograr a través del diálogo que los asesorados se impliquen voluntaria y responsablemente desde la planificación en sus propios procesos formativos, reflexionen sobre lo que ocurre en sus clases, modifiquen sus teorías, desarrollen formas alternativas de actuación, enseñen y permitan aprender con un marcado carácter integrador y desarrollador, y aprendan a vincular lo afectivo y lo cognitivo, lo instructivo y lo educativo.

Por otro lado, el programa que se presenta, se identifica además por favorecer el desarrollo de procesos reflexivos, repensar sobre la práctica y tomar 


\section{Caridad Abreu}

Telos Vol. 21, No. 1 (2019). 114-140

decisiones para generar otro nivel de conocimiento mediante el diálogo, la colaboración y el intercambio entre las partes implicadas (asesor-asesorados), propicia espacios en el cual el sujeto que se supera se considera un investigador de su propia práctica que indaga en sus modos de actuación en el contexto en que se desarrolla, activa a su proceso de formación, y a la toma de decisiones desde el propio contexto.

$\mathrm{Al}$ estar diseñado para preparar a profesores de idioma inglés en la dirección del proceso de enseñanza-aprendizaje de esta disciplina, tiene en cuenta las particularidades del proceso de enseñanza- aprendizaje de la misma, lo que se particulariza en propiciar vías para desarrollar las cuatro habilidades lingüísticas en idioma inglés de forma integral y discursos orales necesarios en el desarrollo del proceso de enseñanza-aprendizaje de esta disciplina.

El programa, además, facilita la dosificación de los contenidos, así como la confección de guías de estudios para ser utilizadas por los estudiantes en la clase encuentro en idioma inglés. Por otro lado, el programa guía al sujeto que se supera en el desarrollo de actividades más allá del encuentro mediante el uso de materiales confeccionados para ser utilizados durante el desarrollo del proceso de enseñanzaaprendizaje y tributa al desarrollo de la independencia cognoscitiva, y a la autonomía en el aprendizaje mediante el desarrollo de estrategias de enseñanza- aprendizaje en idioma inglés.

Las temáticas que se tratan durante su desarrollo parten de tomar en cuenta las necesidades de superación de los docentes y se utilizan textos impresos, materiales digitalizados, tareas evaluativas que se encuentran disponibles en un sitio diseñado para la realización del trabajo independiente de los asesorados.

\section{Principales requisitos del programa de superación profesional para profesores universitarios de idioma inglés.}

En el desarrollo del programa que se propone deben ser tomados en cuenta los siguientes requisitos: 
Se desarrolla desde la perspectiva del asesoramiento colaborativo combinando los momentos individuales y colectivos.

Se caracteriza por la presencia de un asesor (docente que orienta, guía el desarrollo del programa) y los asesorados (sujetos que se superan) donde el primero deberá:

- Desarrollar hábitos y conductas mediante la colaboración.

- Controlar la marcha y la evaluación del proceso (autoevaluación, coevaluación y heteroevaluación).

- Dirigir el trabajo con los profesores más que intervenir sobre ellos.

$>$ Se caracteriza por el diálogo, la colaboración y el intercambio entre las partes implicadas (asesor-asesorados).

$>$ Se identifica por favorecer el desarrollo de procesos reflexivos, repensar sobre la práctica y tomar decisiones para generar otro nivel de conocimiento.

$>$ Se erige de manera flexible para buscar y facilitar la máxima participación e implicación del profesorado dentro del proceso de superación.

$>$ Se identifica por propiciar espacios en el cual el sujeto que se supera se considera un investigador de su propia práctica, que indaga en sus modos de actuación en el contexto en que se desarrolla, de manera que contribuya de forma activa a su proceso de formación, y a la toma de decisiones desde el propio contexto.

Se estructura a partir de tres componentes: El diagnóstico de necesidades, las formas de superación convenidas (talleres y autosuperación) y la evaluación.

$>$ Se desarrolla teniendo en cuenta las particularidades del proceso de enseñanzaaprendizaje de la disciplina Idioma Inglés lo cual lo particulariza al:

- Propiciar el desarrollo de las cuatro habilidades lingüísticas en idioma inglés de forma integral y de discursos orales necesarios en el desarrollo del proceso de enseñanza-aprendizaje de la disciplina Idioma Inglés.

- Orientar la realización del trabajo independiente mediante la reflexión, la metacognición, la toma de decisiones y el uso de las Tecnologías de la Información y las Comunicaciones (TICs) sobre temáticas de interés en los estudiantes.

- Facilitar la dosificación de los contenidos, así como la confección de guías de 


\section{Caridad Abreu}

Telos Vol. 21, No. 1 (2019). 114-140

estudios para los estudiantes para la clase encuentro en idioma inglés.

- Guiar al sujeto que se supera en el desarrollo de actividades más allá del encuentro mediante el uso de materiales confeccionados para ser utilizados durante el proceso de enseñanza-aprendizaje de la disciplina Idioma Inglés.

- Tributar al desarrollo de la independencia cognoscitiva, y a la autonomía en el aprendizaje mediante el desarrollo de estrategias de enseñanza- aprendizaje en idioma inglés.

\section{Componentes del programa de superación profesional para profesores universitarios de idioma inglés.}

El programa de superación profesional para profesores universitarios de idioma Inglés además de contar con elementos constitutivos como la fundamentación, los objetivos, los contenidos, las indicaciones metodológicas y de organización que consideran formas -métodos-medios-tiempo, la evaluación y la bibliografía el programa cuenta además con tres componentes: el diagnóstico de necesidades, las formas de superación convenidas y la evaluación con el fin de orientar al asesor para favorecer procesos que permitan a los asesorados reflexionar sobre su práctica.

El diagnóstico de necesidades, constituye el primer componente del programa. Se parte del análisis de las necesidades de superación de los docentes y se contemplan aspectos como la percepción de los docentes sobre la modalidad semipresencial, la preparación teórico-metodológica de los profesores universitarios de la disciplina Idioma Inglés, así como la efectividad del modo de accionar del docente en la modalidad semipresencial.

Durante el diagnóstico se utilizan diferentes métodos y técnicas como observaciones a clases, observación participante encuestas, así como entrevistas individuales y grupales a profesores de la disciplina Idioma Inglés.

Los datos resultantes se interpretan mediante la triangulación y la conciliación de los resultados lo que permite el establecimiento de necesidades para la preparación de los 
profesores universitarios para dirigir el proceso de enseñanza-aprendizaje de la disciplina Idioma Inglés en la modalidad semipresencial, además, se consideran tanto las debilidades como las potencialidades.

Estos resultados juegan un importante papel en la propuesta, pues, sus presupuestos significan el punto de partida dentro de sus componentes; de modo que el estado de cada uno de los indicadores establecidos puede ser sometidos a comprobación tanto antes, durante, como al concluir la aplicación del programa. Por otro lado, los indicadores permiten proyectar la superación profesional de los docentes mediante el desarrollo de las formas de superación, que constituyen el segundo componente del programa.

Entre las formas de superación, que constituyen el segundo componente del programa se consideran el taller y la auto preparación.

El taller constituye la primera forma de superación convenida y se desarrolla con el objetivo de sistematizar los conocimientos teóricos necesarios a los docentes para dirigir el proceso de enseñanza-aprendizaje en la modalidad semipresencial.

Para el buen funcionamiento de los talleres en el programa de superación se da prioridad al manejo de técnicas grupales como: técnicas de iniciación, de presentación, de producción grupal y otras. Se utilizan además métodos y procedimientos productivos en pos de lograr el desarrollo del pensamiento, la imaginación, la participación, además del diálogo, la discusión socializada, el debate y otros.

Los talleres se desarrollan en correspondencia con el número de temáticas seleccionadas en dependencia de la complejidad de las mismas, los niveles de solución de los problemas presentados, así como la toma de decisiones asumidas.

La forma de evaluación que se utiliza es la autoevaluación, la coevaluación y la heteroevaluación.

Por su parte la auto preparación como segunda forma de superación está presente en cada momento de forma obligatoria y permanente y de su resultado depende que los asesorados puedan resolver las diferentes tareas durante el desarrollo del programa, tanto las que se realizan en cada sesión de trabajo, como durante el estudio 


\section{Caridad Abreu}

Telos Vol. 21, No. 1 (2019). 114-140

independiente.Sus objetivos se orientan hacia el dominio de los contenidos teóricometodológicos fundamentales relacionados con la enseñanza de la disciplina Idioma Inglés en la modalidad semipresencial y sus contenidos se precisan en relación con los objetivos a alcanzar y de acuerdo a las necesidades de superación.

De igual forma se utiliza la autoevaluación, la coevaluación y la heteroevaluación.

El tercer componente del programa lo constituye la evaluación con un carácter integrador, caracterizada por el diálogo, la negociación y la comunicación entre iguales, asesor-asesorado-asesor, lo que posibilita la flexibilidad y la adecuación a partir de las necesidades de los docentes, concepción ésta que se tiene en cuenta a la hora de evaluar.

Desde esta concepción se utilizan fundamentalmente dos formas de evaluación: la diagnóstica y la formativa

La evaluación diagnóstica se precisa en una fase inicial con la finalidad de evaluar las necesidades de preparación de los profesores universitarios para dirigir el proceso de enseñanza-aprendizaje de la disciplina Idioma Inglés en la modalidad semipresencial, mientras que la evaluación formativa se desarrolla durante todo el desarrollo del proceso mediante la autoevaluación, la coevaluación y la heteroevaluación.

Se utilizan además diversas técnicas como diario, además de la realización de observación de la práctica con la utilización de diarios u otros registros sistemáticos.

La evaluación final queda enmarcada fundamentalmente dentro del último taller sobre los resultados obtenidos por los docentes.

\section{Resultados de la aplicación del programa de superación profesional}

Para el análisis de los resultados de la aplicación del programa de superación, se tomaron en cuenta los resultados registrados a partir de las valoraciones hechas por los asesorados, los resultantes de los instrumentos aplicados, así como los resultantes del comportamiento de la preparación de los profesores universitarios para dirigir el proceso de enseñanza- aprendizaje de la disciplina Idioma Inglés en la modalidad 
Programa de superación profesional para profesores universitarios de idioma inglés

semipresencial.

Estos resultados fueron sometidos a un proceso de evaluación a través de la triangulación, lo cual permitió contrastar la información de las diversas fuentes integrando los datos arrojados y situaciones presentadas durante el proceso de investigación, permitiendo determinar las tendencias generales correspondientes a cada categoría o sub- categoría. De esta forma fue posible arribar a los resultados que se presentan a continuación:

Se evidencia un aumento en el desarrollo de los niveles de preparación de los profesores en la dirección del proceso de enseñanza-aprendizaje de la disciplina Idioma Inglés en la modalidad semipresencial a partir de las diferencias significativas observadas resultantes de la evaluación de las categorías y sub-categorías establecidas. Estos resultados son coherentes, pues muestran coincidencia entre los análisis cuantitativos y cualitativos realizados.

La preparación teórico- metodológica recibida por los docentes durante el proceso de superación con la puesta en práctica del programa de superación diseñado les permitió potenciar la reflexión, la metacognición y la toma de decisiones a partir de los conocimientos recibidos durante el desarrollo del programa de superación profesional que se presenta.

Como resultado se evidencia que desde el inicio algunas categorías y subcategorías no eran favorables, y estas se traducían de igual forma a escala procedimental, sobre todo por estar visiblemente afectados en su dimensión cognitiva, y afectiva, sin embargo, esta situación se fue transformando de manera positiva a medida que transcurría el proceso de superación el desarrollo del programa de superación profesional que se presenta.

Los resultados obtenidos demuestran transformaciones en los docentes que conforman la muestra tanto en lo conceptual como en lo procedimental. Lo anterior se pudo comprobar mediante las nuevas concepciones, y opiniones expresadas como resultado de un proceso de reflexión lo que demuestra un nivel superior en la preparación teórico-metodológica que realizan. 


\section{Conclusiones}

La aplicación de la modalidad semipresencial ha representado un gran reto y un desafío para los profesores universitarios de la disciplina Idioma Inglés dadas las características del proceso de enseñanza-aprendizaje de la disciplina que imparten, y la ausencia de una experiencia anterior en la enseñanza del idioma inglés con una modalidad semipresencial.

La superación profesional del profesor universitario de la disciplina Idioma Inglés se presenta como una necesidad y una alternativa viable para lograr la preparación teórico-metodológica necesaria para dirigir el proceso de enseñanzaaprendizaje de esta disciplina en la modalidad semipresencial.

La superación del docente para dirigir el proceso de enseñanza-aprendizaje en correspondencia con las exigencias de la modalidad semipresencial debe ser organizada a través de formas de superación orientadas al perfeccionamiento del modo de accionar del docente a partir de sus vivencias y así permitir la elaboración del conocimiento, la reflexión y el análisis mediado por la colaboración y la toma de decisiones.

El Programa de Superación Profesional que se presenta se distingue por asumir el asesoramiento colaborativo como forma de superación para lograr la preparación del profesor universitario de Idioma Inglés en la dirección del proceso de enseñanzaaprendizaje de la disciplina inglés en la modalidad semipresencial

El proceso de enseñanza- aprendizaje en el programa se fundamenta en la teoría Histórico- Cultural, donde se asume a la enseñanza como guía al desarrollo, y el proceso transcurre en un grupo en el cual se propician múltiples relaciones sociales, y se enriquecen, produciéndose nuevas necesidades, conocimientos, experiencias, lo que obviamente no implica desconocer las particularidades de los integrantes

El programa cuenta con elementos constitutivos como la fundamentación, los objetivos, los contenidos, las indicaciones metodológicas y de organización que consideran formas -métodos-medios-tiempo, la evaluación y la bibliografía. 
El programa se caracteriza por contar además con tres componentes: el diagnóstico de necesidades, las formas de superación convenidas y la evaluación con el fin de orientar al asesor para favorecer procesos que permitan a los asesorados reflexionar sobre su práctica.

El Programa de Superación profesional que se presenta se distingue por asumir el asesoramiento colaborativo como forma de superación profesional y se caracteriza por erigirse de forma flexible , buscar y facilitar la máxima participación e implicación del profesorado, establecer un clima relacional y un tono afectivo, favorecer la toma de decisiones así como el desarrollo de procesos que permitan a los docentes reflexionar sobre su práctica, y repensar la práctica para generar otro nivel de conocimiento.

\section{Referencias Bibliográficas.}

Abreu, Caridad. (2007). Intervención psicopedagógica a favor del aprendizaje de la comunicación oral en idioma inglés. II Taller Regional de Educación en Ciencias de la Salud. (ISCMVC) Santa Clara. Cuba.

Abreu, Caridad. (2008). La superación profesional del profesor universitario de la disciplina Idioma Inglés para dirigir el Proceso de Enseñanza-Aprendizaje en la modalidad semipresencial. Tesis en opción al grado científico de Doctor en Ciencias Pedagógicas.Universidad Central "Marta Abreu" de Las Villas. Cuba.

Addine, Fátima. (2002): Los principios de la dirección del Proceso Pedagógico. Compendio de Pedagogía. Editorial Pueblo y Educación. La Habana.

Alonso, Freyre Joaquín; Rivero Pino, Ramón y Riera Vázquez, Celia (2004) El autodesarrollo comunitario (Primera edición). Editorial Feijoo. Cuba

Álvarez de Zayas, Carlos. (1999). La escuela en la vida. Educación Superior (3era Edición) Editorial Pueblo y Educación. Cuba.

Añorga, Julia. (1997). Educación avanzada y producción de conocimientos en América Latina. CENESEDA, ISPEJV, La Habana. En: Morles, Víctor (Comp.): Postgrado y desarrollo en América Latina. vol.4.Editorial CEISEA.Venezuela.

Arroyo, Miguel (1999) Ciclos de desenvolvimento humano e formação de educadores. Educação\&Sociedade, v. 20, n. 68, Brasil (Pp.143-161). 
Arroyo, Miguel (2001): La superación profesional de los profesores universitarios en la disciplina idioma inglés: su perfeccionamiento y aplicación en la Universidad de Pinar del Río. Tesis presentada en opción al grado científico de Doctor en Ciencias Pedagógicas. Cuba.

Berges, Maritza. (2003): Modelo de Superación Profesional para el Perfeccionamiento de Habilidades Comunicativas en Docentes de la Secundaria Básica. Tesis en opción al grado de Doctora en Ciencias Pedagógicas. Cuba.

Carretero, María Reyes. (2002). El asesoramiento psicopedagógico. Tesis en opción al título de Doctor en Ciencias Pedagógicas. Universidad de Girona. España.

Corona, Dolores. (2006). La enseñanza del idioma inglés en la universidad cubana a inicios del siglo XXI. Reflexiones en el 40 Aniversario de la Reforma Universitaria. Revista Cubana de Educación Superior. Vol. XXI (3) 2001. Cuba. (Pp. 29-37)

Escudero, Juan Manuel y Moreno, Juan Manuel (1992). El asesoramiento a centros educativos. Editorial: Consejería de Cultura y Educación. España

Freire, Paulo(1996)Pedagogia da Autonomia:saberes necessários à prática educativa/Editorial.Paz e Terra. Brasil

Gallardo, Teresita. (2004). La Educación en Valores Morales en el Contexto Empresarial a partir del Vínculo Universidad-Empresa: Hacia un modelo de Superación a Directivos. Tesis en opción al grado de Doctora en Ciencias Pedagógicas. Cuba.

Gómez Anoceto, Oscar (2007): Estudio de la Cultura Física en la zona de montaña de la región Central de Cuba: Propuesta de un modelo de intervención. Tesis en opción del grado científico de Doctor en Ciencias Pedagógicas. ISCFVC "Manuel Fajardo." Cuba.

González Morales, Alfredo (1999) El método de la modelación: su aplicación en las ciencias pedagógicas. Material Impreso. Cuba.

González Rey, Fernando. (1989). La personalidad, su educación y desarrollo. Pueblo y educación. Cuba.

Halliday, Michael (1982). Exploraciones sobre las funciones del lenguaje. Editorial Médica y Técnica, S.A. España.

Lombana, Raúl. (2005). La superación profesional con enfoque interdisciplinario en el docente de humanidades de la escuela de instructores de arte. Tesis presentada para optar por el grado científico de Doctor en Ciencias Pedagógicas. UCLV. Cuba. 
López, Yanes. (1997). Asesoramiento curricular y organizativo en educación Editorial: Ariel Educación. España.

Martínez, Miguelez Miguel (1997) El paradigma emergente: Hacia una nueva teoría de la racionalidad científica. Editorial Trillas. México

Monereo Carlos y Pozo Juan Ignacio (2005). La práctica del asesoramiento educativo a examen. Editorial Graó. España

Nieto, Miguel José. (2005). El asesoramiento pedagógico a centros escolares. Revisión teórica y estudio de casos. Tesis doctoral inédita, Universidad de Murcia. España.

Lagardera Otero, Francisco (1998) Sobre el objeto de estudo y metodología de las ciencias de la actividad deportiva. Revista Educación Física e deporte no seculo XXI. Vol1, n1. España (Pp. 81-86)

Peralta, Castellón Yanelys (2006) Programa de Asesoramiento Psicopedagógico en evaluación del aprendizaje para profesores en la universalización de la Educación. Tesis en opción al grado de Doctora en Ciencias Pedagógicas. Cuba.

Pérez Serrano, Gloria (1999). Investigación cualitativa: retos e Interrogantes, Técnicas y análisis. Editora Muralla. España

Rodríguez Gómez, Gregorio; Gil Flores, Javier y Jiménez García, Eduardo (2004) Metodología de la investigación cualitativa. (1era. ed.) Editorial Félix Varela. Cuba.

Romero, Claudia. (2007). Gestión del Conocimiento, Asesoramiento Mejora Escolar. "El caso de la escalera vacía". Profesorado. Revista de currículum y formación del profesorado. vol11, n1. Argentina (Pp1-23)

Sánchez, María Rita. (2013). La supervisión como estrategia de formación de profesores mentores: Una aplicación práctica. Revista Innovación Educativa. Vol. 3, n. 1. México (Pp17-26).

Torres, Carlos Alberto (2004) Educación, poder y biografía, Diálogos con educadores críticos. Perfiles educativos. vol.28 n.(112) México (Pp.301)

Vygotsky, Lev (1987): El desarrollo de las funciones síquicas superiores. Editorial Científica Técnica. Cuba

Vygotsky, Lev (1978). Mind in society: The development of higher psychological processes Cambridge, Mass.: Harvard University Press. England 